\title{
FACT-CHECKING \& SOCIAL MEDIA SHARING BEHAVIOR AMONG UAE YOUTH
}

\author{
Ashraf Khalila ${ }^{\mathrm{a}}$, Salam Abdallah ${ }^{\mathrm{b}}$ and Kundan Noor Sheikh ${ }^{1}$ \\ ${ }^{a}$ Department of Computer Science and Information Technology, Abu Dhabi University \\ Abu Dhabi, United Arab Emirates \\ ${ }^{b}$ College of Business, Abu Dhabi University \\ Abu Dhabi, United Arab Emirates
}

\begin{abstract}
With the advent of the internet and social media, the spread of online misinformation has become a matter of serious concern for scholars and decision makers all over the world. Given this platform's relative lack of censorship and extensive as well as swift reach, the likelihood of unverified information content spreading unchallenged in the digital world has grown exponentially. This study aims to understand the patterns of content-sharing behavior on major social media platforms by young adults living in United Arab Emirates, and how this is influenced by various demographic and social factors in an individual user's life, such as social conformity and religiosity. A qualitative analysis of 6 semi-structured interviews on young adult users ranging between 18 to 24 years of age in the United Arab Emirates (UAE) was conducted to ascertain their sharing patterns as well as tendencies to verify content being shared via social media. The study finds that the need for social conformity and religiosity influence what users share on social media and how likely they are to verify information before sharing it.
\end{abstract}

\section{KEYWORDS}

Social Media, Online Misinformation, Privacy, Young Adult, Religiosity, Social Conformity

\section{INTRODUCTION}

Limited censorship in social media has led to unprecedented sharing of unverified content such as rumors and fake news. Hence recent works in the field of Human-Computer Interaction (HCI) have extensively studied sharing practices on social media and how the veracity of information being shared is a growing concern. Starbird, et al. (2016) have identified and described types of 'expressed uncertainty' in social media posts on Twitter during different points in a rumor's lifecycle, across different rumors, and within multiple crisis events. Using Artificial Intelligence (AI) nudges for fact-checking to influence human and machine behavior in an effort to make it more pro-social while preserving individual liberty has also been explored. (Matias, 2017) The effect of reciprocity in sharing behavior and engagement practices is also seen to have significant importance. (Grinberg, et al., 2016) Therefore, disambiguating the "social" aspect of sharing behavior is considered to be of prime importance in order to ensure safe sharing practices whereby content and information being shared and re-shared by young adult users is true and authentic.

Given its unique background and demographic composition, the UAE plays a pivotal and interesting role in the region with regards to content-sharing via social media, its importance and its impact. Despite being one of the smallest Middle Eastern nations in terms of population, the UAE is an Internet adoption leader in the Arab world, with a fast-growing base of internet users. Overall, in the Arab world, the media environment has started opening up recently, especially with social media platforms providing increased opportunities for self-expression and information sharing. (Wiest \& Eltantawy, 2015) However, due to the socio-cultural values of most users in this region, concerns for privacy and reliability of information-sharing have been paramount. An initial study on the use of social media in UAE pointed out that overall users can be said to have a "guarded trust" of the media, especially for media they feel operate correctly and protect their privacy.

${ }^{1}$ kundanrulz@gmail.com 
(Al-jenaibi, 2015) Exploring the meaning of "privacy" from the perspective of Qatari nationals, Abokhodair, et al. (2016) have concluded that in the Arab Gulf, the need for privacy is often supported by Quranic text, and privacy is framed as a communal attribute, including not only the individual, but the behavior of those around them; it even extends beyond one's lifespan. Such previously unexplored interpretations of privacy play a role in how users' social, cultural and religious contexts play a major role in how they navigate social media.

Hence, set against this backdrop cultural uniqueness and demographic diversity in the region, young adults in UAE are definitely likely to take into account certain socio-cultural factors when deciding not only what content to share on social media, but also how to verify the authenticity of what is being shared as they feel a high sense of social responsibility towards those who will interact with this shared content.

The aim of this research is to help identify a new angle of sharing habits relevant to this region in particular, where religion and culture is such an integral part of social interaction.

\subsection{Religiosity}

The terms religiosity is often defined as an individual's conviction, devotion, and veneration towards a particular religion or theology. In its most comprehensive use, religiosity can encapsulate all dimensions of religion, gauged by an involvement in religious practices and how much influence one's religious beliefs have on one's day-to-day dealings in life. (Gallagher \& Tierney, 2013)

Religiosity has been used as a dominant factor in social sciences to determine patterns of religious influences among American adolescents. (Smith, 2003) Several reliable studies show that youth and their families who regularly participate in religious activities and rituals display greater overall satisfaction with their lives and feel better equipped to solve health-related problems than those who don't engage in such activities as often. A number of studies also show that religion is associated with more effective "coping" with problems by youth, among teens and young adults. (Shortz \& Worthington, 1994; Varon \& Riley, 1999)

A 10-item intrinsic Religious Motivation Scale proposed by Hoge (1972) with slight modifications to adjust for cultural norms in the Arab world (Abdel - Khalek, 2017).

\subsection{Social Conformity}

In order to identify the level of social conformity in our participants, our online survey took them through a series of statements to agree or diagree with using a likert scale. This was done using a Concern for Appropriateness Scale (CAS) developed by Lennox and Wolfe in 1984, which was validated fully by Martin A Johnson in 1989. (Johnson, 1989)

Melman, et al. (2009) have verified the applicability of the Hebrew version of CAS internationally beyond the Western culture recommending its use in the fields of social psychology, employment, business and personality research. Thus it was appropriate for us to apply it to the UAE population with slight cultural adjustments as suggested by the authors of this study.

Bliss \& Brown (1994) have used Lennox and Wolfe's CAS to investigate the direct and indirect relationship between social conformity (representative of peer pressure, self-representation, etc.) and the use of alcohol and marijuana among 143 American college students. Not only did this scale directly predict Marijuana use, but also interacted with religiosity in the prediction of marijuana use. As for alcohol use, CAS did not directly predict alcohol use but this scale interacted with religiosity and gender in in the prediction of alcohol use. Thus proving the validity of the CAS as an index of social anxiety among young adults leading to specific behavior patterns just to 'fit in'.

We have thus applied similar principles in our study to find the correlation between CAS and patterns of sharing and verifying shared content on social media, as well as the interaction of CAS with religiosity (represented by the HOGE scale explained above) and the demographics of gender among our sample of young adults living in the UAE. 


\subsection{Personality}

A brief 10-item measure of the Big-Five personality dimensions was used to determine the personality traits of our participants in the quantitative study along the scales of extraversion vs. introversion, openness vs. closedness to experience, conscientiousness vs. lack of direction, agreeableness vs. antagonism and neuroticism vs. emotional stability. (Gosling, et al., 2003; John \& Srivastava, 1999) This scale was used to determine how these personality traits affect sharing behavior among participants directly and indirectly through social conformity.

\section{BODY OF PAPER}

Our study focuses on three main research objectives:

1. Identifying social, cultural and religious factors that influence the type of content that young adults share on social media

2. Determining the influence of these factors on the type of content shared

3. Determining what role this influence plays in the likelihood of users verifying the content before sharing it - and if so, how do they verify it?

To answer the above questions, we conducted an in-depth qualitative analysis into the motivations behind social media sharing behavior among young adults in the UAE, and what measures they took to verify the veracity of this information. Data were derived from young adults of various nationalities aged between 18 to 24 years residing within the UAE. Six face-to-face, semi-structured, in-depth interviews were conducted for this qualitative analysis. Before starting the interview, each participant was made to fill out an online survey designed using the Big Five Personality Traits, HOGE Scale for Religiosity (Hoge, et al., 1982) and Concern for Appropriateness Scale (CAS) for social conformity in order to determine the linkages between their score on these scales and their interview answers. (Lennox \& Wolfe, 1984; Johnson, 1989)

\subsection{Social Media Sharing and Verification Behavior}

Six in-depth interviews, each longer than 20 minutes, were conducted. All participants were young adult students residing in the UAE. Three of them identified as male and three as female. All of them identified as Muslim. Research conducted by Dworkin (2012) was taken into consideration when deciding this sample size, whereby "An extremely large number of articles, book chapters, and books recommend guidance and suggest anywhere from 5 to 50 participants as adequate" for qualitative analyses using in-depth interviews.

The design of the interviews was semi-structured, whereby the focus was to get a general overview of participants sharing habits, mainly what type of content they share, what motivates them to share it and how they feel about the importance of credibility of information being shared through social media, especially sensitive content like religious quotes, breaking news, etc. Based on these detailed open-ended discussions, the following themes were extracted regarding the participants' sharing behavior on social media which give insights into their need for social conformity when sharing content as well as their motivations and ideas behind making the online information-sharing space a safe one.

\subsubsection{Empathy and Concern for the Collective Value System}

Empathy and concern for how their post makes others feel, emerged as a recurring theme throughout this study, as one participant mentioned upon being questioned about her motivations behind sharing sayings and inspirational stories on social media, "It's essentially just to spread the goodness." followed by "why spread the hate" when the discussion flowed into whether or not she would be inclined to share controversial religious content through her profile on Facebook.

Furthermore, participants in our study showed a keen sense of respecting collective beliefs and values prevalent in the society around them, acknowledging the fact that the same value system extends into their interactions and sharing habits on social media as well, when one participant observes, "Values shape everything about us...so if there is something that might be against my values or the values of my friends or family, for example, I wouldn't be sending it around or having a look at it myself even. So for example, some 
hate videos come up on Facebook against some religions and ethnicities, I don't even bother looking at them, because then I'm just wasting my time over things that are not worth my time." Another user, when questioned about how he feels regarding his friends and acquaintances on his social network sharing unverified religious content, stated, "Well, I stop paying attention to their posts in the future. I just ignore their posts. Their credibility goes down. For example, there are religious views, like a hadith or any saying from the Prophet Mohammed, which you are quoting in your status, then you NEED to write where it is taken from. It is very clear that we need to prevent a lot of unnecessary and false information from entering into our value system and our social beliefs".

\subsubsection{Importance of Social Conformity and Preserving Self-Image}

Another trend that was observed was the participants' unwillingness to debunk information publically on social media as "everyone's watching". In this context, when asked about their inclination to openly negate false information shared by friends on social media by, for example, commenting on such a post, one participant observed, "I won't really say anything to them by commenting publicly on Facebook, for example...but if we ever have a discussion about it face-to-face later, I would give my opinion. But otherwise, I won't oppose them on social media platform. I try to steer clear of getting involved in controversial issues on social media. Better to play it safe than be rude and offend someone you are friends with."

Another comment that made the concern for self-image fairly evident was, "I am very careful about what I post, as I don't want unsolicited, possibly incorrect, information to spread through me. I also feel that if I am not an authority on the subject, then I am not the right person to be sharing it. Like, for example, there are lots of people nowadays who share things after reading, say, just 'one' news article about it without any background knowledge about the subject matter. So I feel that is very wrong and extremely unfair to those you are sharing it with. So, for me, if I am not an expert on a particular topic, then maybe I don't have the right to post about it, you know."

\subsubsection{Moral Responsibility towards Correcting the Information Space}

Examining how users correct online rumors on Twitter, Arif, et al. (2017) uncovered three different objectives for taking correcting actions: correcting oneself, correcting the information space, and correcting another person (or organization). A deeper analysis was undertaken to identify users' rationale for their corrective actions in relation to these three correction objectives. Consequently several perspectives on the "locus of responsibility" for sharing and correcting rumors were identified, whereby when a user take the responsibility of correcting a rumor upon herself, she is most likely to correct her own previous post upon finding out the truth, while if a user presumes the responsibility of rumor-correcting upon the crowd accessing the information or the social media platform itself, then the likelihood of her correcting her own post after it has been debunked goes down. (Arif, et al., 2017)

A recurring concern participants in our study expressed was regarding the veracity of information being shared via social media and how checks and balance need to be incorporated by users themselves. None of the interviewees were of the opinion that unverified information should ever be shared on social media and most of them agreed that the onus of checking for its authenticity before sharing lies upon the users themselves. They expressed displeasure upon seeing inauthentic content being shared by their friends and acquaintances on social media, and went on to suggest ways of verifying sensitive content before sharing it, so as to cleanse the online social media sharing space of plagues such as fake news and misinformation. Expressing her concern for such instances, one participant said, "When people usually send these kind of videos with stats in them, a lot of times stats are actually exaggerated because that person just wants to drive their point home... so like instead of saying only about 50 people were affected, they'll say something like two-thirds of the population was affected, which is kind of an exaggerated way of saying just 50 people were affected...maybe because 50 sounds like a small number in comparison to the billions that we have on this planet. So, things like these, because they're usually exaggerated, I don't tend to go for them because I don't know if they're true or not...or how much they have been twisted just to drive a point across..."

On further probing about how such misinformation can be corrected, a sense of moral obligation was expressed, "let's say there's a blogger saying things against a certain minority, like some kind of hate message, you can't really stop them directly. But what you can do is stop spreading it if it comes up to you...prevent it from reaching others and stop the hate from spreading further". So there was a general 
resolve to not re-share information that has not been proven to be correct, so as to prevent the wrong from spreading.

The participants also shared details about their own ways of checking the authenticity of content they come across on social media, with one of them commenting, "whether I believe what I read or not, depends on the source. So if its let's say CNN, I'm more inclined to believe it... if its Huffington post, I would believe it. But is it's just a normal random page, like a blog or something...then I wouldn't believe it right away." Another participant added a similar view saying, "For me, if it's coming from a reliable source like Khaleej times or Gulf news, so I would consider that authentic. And they update it. So if I go to the website of Khaleej times and I am following a story, so I can get all the updates from it. So if at any point in time, the information is proven to be wrong, I can update it, and tell other people about it as well. But usually people don't do that, which is something I dislike. I feel we should keep a constant check on what we are posting on social media, so we can keep a track of whether it is right or wrong. It is our responsibility to share the truth."

As mentioned before, most participants were inclined towards correcting the online information space tacitly rather publicly, so when commenting on the measures taken on their part when their friends post controversial or unverified content, one of them said, "Mainly, I STOP trusting people who share such unverified content... because you see I don't tend to blindly share stuff just because everyone is doing it, but when and if I do share something, then it means that I have done some background research and I have fair knowledge about the subject matter...but I know that other people do not follow the same principles. They share things without checking, without verifying and end up spreading wrong information left, right and center..." when further probed about how this makes him feel, he added, "it really upsets me... I think that on a personal level, people should be aware of the fact that whatever they share should be verified because, in general, I'm sure no one is in favor of our future generations being exposed to knowledge and information which is misleading."

Discussing measures that can be taken to prevent such misinformation from spreading, he added, "So we should take the responsibility to correct this on our own. But if people are still not abiding by such basic values and principles, then perhaps awareness campaigns should be a thing. I know that it sounds like a rather trivial issue to be campaigning for, but it is extremely important at the same time. I mean, its free space, right? Anyone and everyone can post pretty much whatever they like, and that alone, comes with a sense of responsibility...so what's the difference between someone people who deliberately WANT to spread rumors and those who are doing it simply out of ignorance because they fail to 'check' before they post something. In the cyberspace, they're both equally wrong...there's no excuse for it! So we have to teach our youth where to draw the line. And for that I feel that awareness campaigns are a good idea...one of the better solutions to the problem. It is also a good idea to start it from our younger generation, since they are at the forefront and tend to use these platforms most frequently... So I think we should start awareness sessions in schools, colleges and then universities to introduce the idea of ethical sharing."

Mentioning people who misquote religious content on social media in specific, he further added, "I think we should minimize this by quoting the credentials and...that's something at we are being taught regularly even at university, you know...the importance of citation... whatever you write in your assignments, reports and research papers should be verified. Same principle applies, you know. Why blindly copy-pasted things without fair background knowledge? It makes sense, basically, to research a little on something that you are so confidently sharing on social media with hundreds and thousands of people...verify it, and cite the source where possible...else, steer clear!"

\section{CONCLUSION}

The qualitative analysis of our participants' sharing behavior proved that a need for social conformity and religiosity play a major role in determining the content that is being shared by young adults in the UAE. It also influences their decision to verify content being shared, especially sensitive information concerning religious beliefs, breaking news, etc. A sense of moral responsibility towards cleansing the information space to benefit other users and to save them from harm was also seen.

Based on our qualitative study, we can conclude that young adults in the UAE experience a collective sense of empathy and concern for the value system prevalent in the region. This was partially pointed out by Wiest \& Eltantawy (2015) and has been proven further in a more multi-faceted context through our current 
study. In order to preserve 'peace' within their relations and bonds developing via social media, these youngsters were opposed to the idea of sharing controversial content against any religion or ethnicity, so as to not offend anyone in the diverse socio-cultural context of their region, just as they do in real life. Their primary objective for sharing content on social media was to spread goodness rather than negativity and to be a source of helping others network rather than harming them.

Secondly, not only are the youth concerned about conforming to the social, moral and cultural values of their surroundings when engaging with content on social media, they are also significantly concerned about their self-image when sharing, re-sharing or engaging with such content. This inculcates a high level of conscientious sharing behavior, whereby these users are generally very careful about what they post/share and always try their best to verify the source of any sensitive content they may be sharing, be it religious text or breaking news. The downside to this need for preserving self-image is that they are less likely to openly publicly debunk false information (such as fake news) being shared by their own family, friends and acquaintances on social media, as a high percentage of users interviewed expressed hesitation in making an effort to publicly correct their friends for posting wrong/controversial material on social media. So, for example, instead of commenting on a post saying "this is fake news", they would rather tell the original poster in private if they are close enough or just ignore it if they don't the personal too closely. However, they all agreed that such posters do lose their future credibility, and that information shared through such people would always be received with a grain of salt, i.e. being on the receiving end of such content, our young adults would always think twice about believing it without verifying on their own through other reliable sources. So 'trust but verify'!

Lastly, our participants were of the opinion that the onus of responsibility for verifying information shared via social media falls upon the user herself. Hence they showed a strong conviction/commitment towards always verifying sensitive information against various credible sources before posting it on their social media. They also displayed a certain level of resistance towards blindly believing anything posted by their friends on social media without verifying it for themselves, as they felt it was their own responsibility to consume/receive authentic information - be morally responsible consumers and sharers/posters of knowledge/information.

Future research will develop measurement instruments based on the findings derived from this research. These measurement instruments will provide us a better means for validating our hypothesis and testing for control and mediating variables.

\section{REFERENCES}

Abdel - Khalek, A. M., 2017. The Construction and Validation of the Arabic Scale of Intrinsic Religiosity (ASIR). Psychol Behavioral Science International Journal, Vol. 44, No. 4.

Abokhodair, N. et al., 2016. Privacy and Twitter in Qatar: Traditional Values in the Digital World. Proceedings of the 2016 ACM Web Science Conference. New york, USA, pp. 66-77.

Al-jenaibi, B., 2015. Use of Social Media in the United Arab Emirates: An Initial Study. Global Media Journal, Vol. 1, No. 2, pp. 3-27.

Arif, A. et al., 2017. A Closer Look at the Self-Correcting Crowd: Examining Corrections in Online Rumors. Proceedings of the 2017 ACM Conference on Computer Supported Cooperative Work. Portland, USA, pp. 155-168.

Bliss, S. K., \& Brown, C. L., 1994. Concern for Appropriateness, Religiosity, and gender as predictors of Alcohol and Marijuana use. Social Behavior and Personality, Vol. 22, No. 3, pp. 227-238.

Dworkin, S. L., 2012. Sample Size Policy for Qualitative Studies Using In-Depth Interviews. Archives of Sexual Behavior, Vol. 41, No. 6, pp. 1319-1320.

Gallagher, S., \& Tierney, W., 2013. Religiosity/Religiousness. In: M. Gellman, \& J. Turner, Encyclopedia of Behavioral Medicine. Springer, New York, USA, pp. 108.

Gosling, S. D. et al., 2003. A very brief measure of the Big-Five personality domains. Journal of Research in Personality, Vol. 37, pp. 504-528.

Grinberg, N., et al., 2016. Changes in Engagement Before and After Posting to Facebook. Proceedings of the 2016 CHI Conference on Human Factors in Computing Systems, San Jose, USA, pp. 564-574.

Hoge, D. R., 1972. A Validated Intrinsic Religious Motivation Scale. Journal for the Scientific Study of Religion, Vol. 11, No. 4, pp. 369-376. 
Hoge, D. R., et al., 1982. Transmission of Religious and Social Values from Parents to Teenage Children. Journal of Marriage and Family, Vol. 44, No. 3, pp. 569-580.

John, P. O., \& Srivastava, S., 1999. The Big-Five Trait Taxonomy: History, Measurement and Theoretical Perspectives. Handbook of personality: Theory and research, pp. 22-34.

Johnson, M. A., 1989. Concern for Appropriateness Scale and Behavioral Conformity. Journal of Personality Assessment, Vol. 53, No. 3, pp. 567-574.

Lennox, R., \& Wolfe, R. N., 1984. Revision of the Self-Monitoring Scale. Journal of personality and social psychology, Vol. 46, No. 6, pp. 1349-64.

Matias, J. N., 2017. MIT Media Lab (via medium.com). Retrieved January 3, 2018, from https://medium.com/mit-medialab/persuading-algorithms-with-an-ai-nudge-25c92293df1d\#.y62422rs5

Melman, R. B., et al., 2009. The Psychometric Properties of the RSMS and CAS in Hebrew. European Journal of Psychological Assessment, Vol. 25, pp. 8-15.

Shortz, J., \& Worthington, E., 1994. Young adults' recall of religiosity, attributions, and coping in parental divorce. Journal for the Scientific Study of Religion, Vol. 33, pp. 172-179.

Smith, C., 2003. Theorizing Religious Effects Among American Adolescents. Journal for the Scientific Study of Religion, Vol. 42, No. 1, pp. 17-30.

Starbird, K., et al., 2016. Could This Be True? I Think So! Expressed Uncertainty in Online Rumoring. Proceedings of the 2016 Conference on Human Factors in Computing Systems, San Jose, USA, pp. 360-371.

Varon, S., \& Riley, A., 1999. Relationship between maternal church attendance and adolescent mental health and social functioning. Psychiatric Services, Vol. 50, pp. 799-805.

Wiest, J. B., \& Eltantawy, N., 2015. Mediatization in the Arab World: A Cross-cultural comparison of New Media use. Online Journal of Communication and Media Technology, Vol. 5, pp. 120-142. 